\title{
Real-Space Evidence of Rare Guanine Tautomer Induced by Water
}

\section{Supporting Information}

\author{
Chi Zhang ${ }^{\dagger}$, Lei Xie ${ }^{\dagger}$, Yuanqi Ding, Qiang Sun, Wei Xu* \\ Tongji-Aarhus Joint Research Center for Nanostructures and Functional Nanomaterials, College \\ of Materials Science and Engineering, Tongji University, Caoan Road 4800, Shanghai 201804, \\ People's Republic of China. \\ E-mail: xuwei@tongji.edu.cn
}




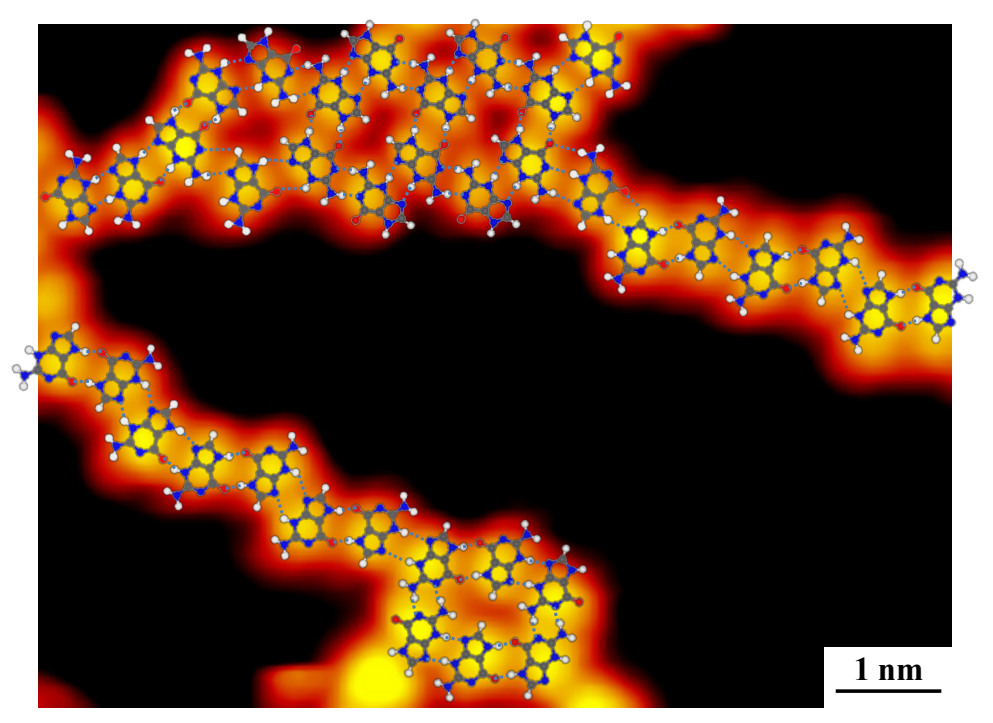

Figure S1. STM image showing the formation of chain structures together with six-membered ring structure superimposed with DFT optimized structural models where the chain structures are usually part of the ring structure with the same characteristic of hydrogen bonds.

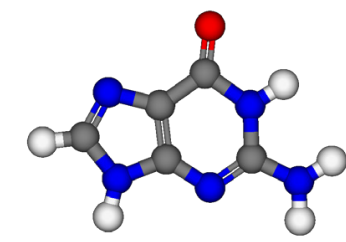

$(1,9)$

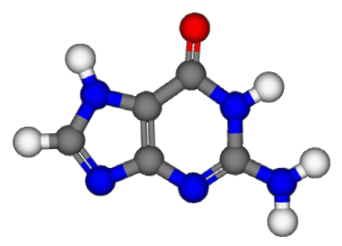

$(1,7)$

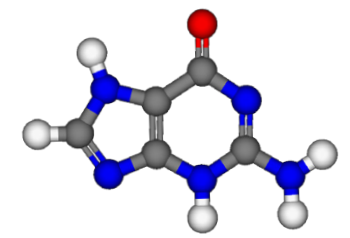

$(3,7)$



$(7, \mathbf{O} 2)$

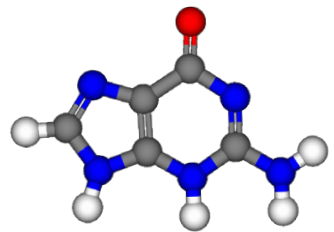

$(3,9)$

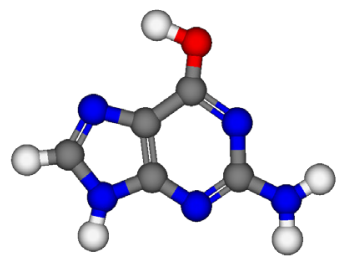

$(9,01)$

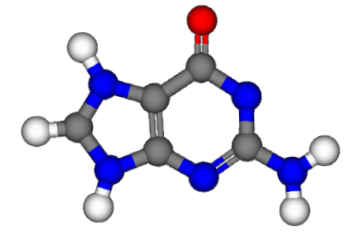

$(7,9)$

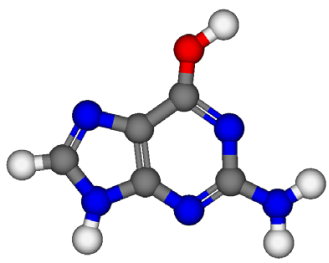

$(9, \mathbf{O} 2)$

Figure S2. The eight stable G tautomers considered in the theoretical study. ${ }^{1}$ 


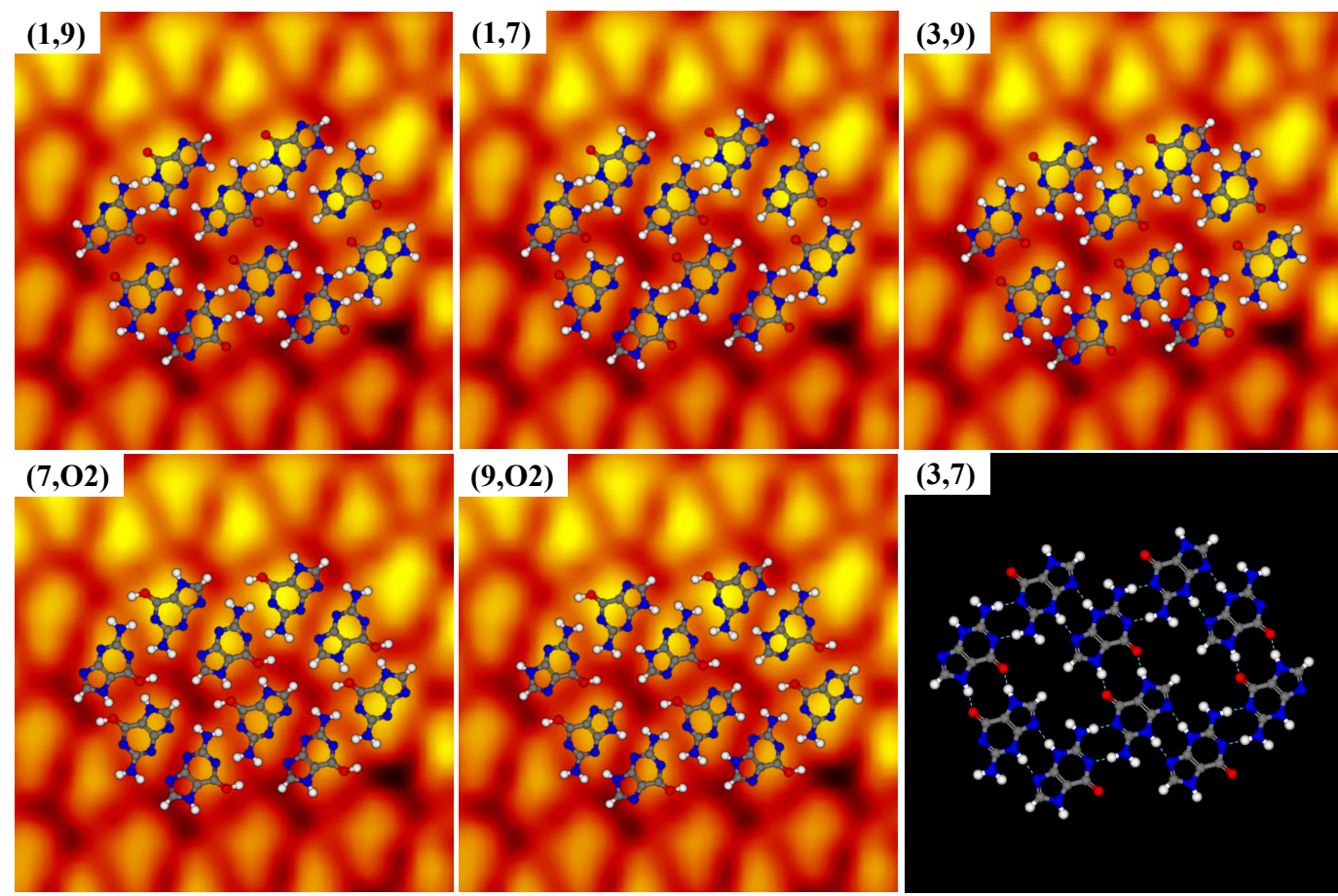

Figure S3. STM images of typical water-induced network structure superimposed with $(1,9),(1,7)$, $(3,9),(7, \mathrm{O} 2)$ and $(9, \mathrm{O} 2)$ tautomeric forms, in which none of these forms can well account for the observed structure with reasonable intermolecular hydrogen bonds, and these forms are thus ruled out. The $(3,7)$ network structure is shown beside as a reference.

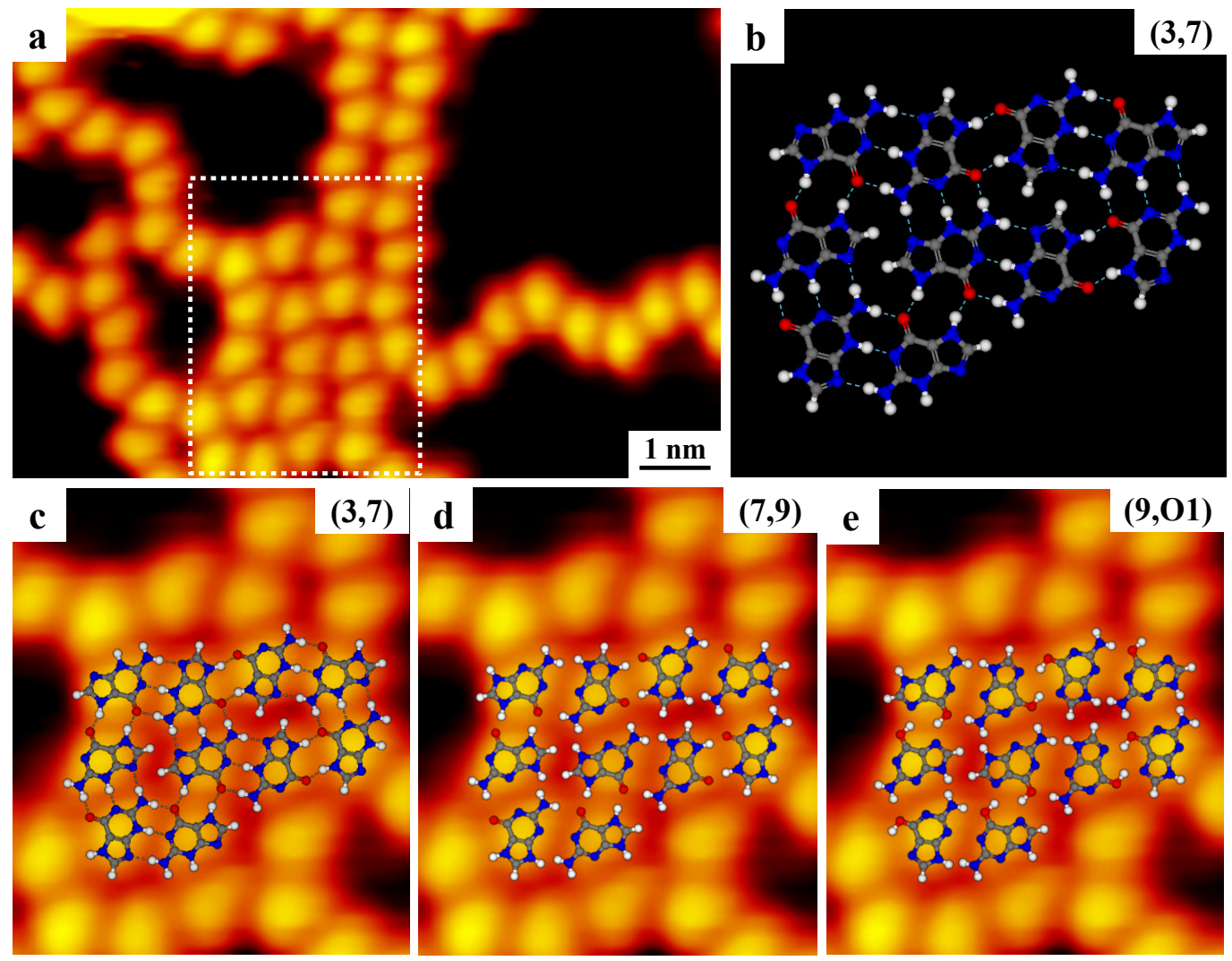


Figure S4. STM images of some different nodes coexisting with the parallel six-membered rings superimposed with $(3,7),(7,9),(9, \mathrm{O} 1)$ tautomeric forms. (a) STM image of some different nodes coexisting with the parallel six-membered rings after exposing the G-precovered surface to the water atmosphere or direct deposition of $\mathrm{G}$ in the water atmosphere. (b) The corresponding DFT-optimized structural model composed of $\mathrm{G} /(3 \mathrm{H}, 7 \mathrm{H})$. (c) The zoom-in STM image of the nodes marked by rectangle contour in (a) superimposed by the structural model of $\mathrm{G} /(3 \mathrm{H}, 7 \mathrm{H})$ form with a good agreement. (e) and (f) STM images of the nodes superimposed by $(7,9)$ and $(9, \mathrm{O} 1)$ forms where none of them can well account for the observed structure with reasonable intermolecular hydrogen bonds, and these forms are thus ruled out.

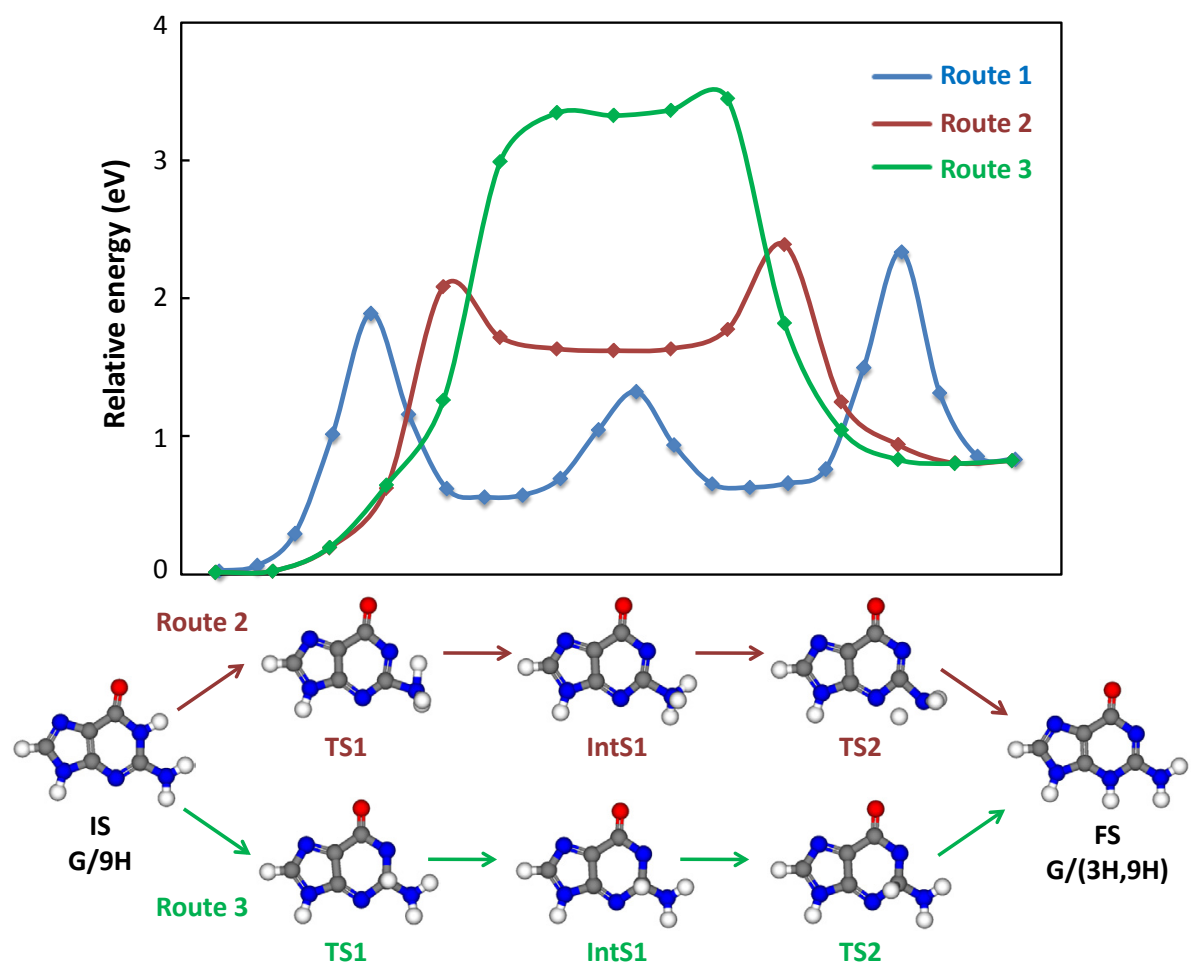

Figure S5. DFT calculated three reaction pathways for the first-step tautomerization from G/9H to $\mathrm{G} /(3 \mathrm{H}, 9 \mathrm{H})$ in the gas phase involving a proton-transfer process from $\mathrm{N} 1$ to $\mathrm{N} 3$ where route 1 is found to be energetically most favorable and shown in the main text in Figure 3. The structural models of the local minima (IS, IntS1 and FS) and transition states (TS1 and TS2) along different reaction paths (Route 2 and Route 3) are shown below the energy diagrams. 


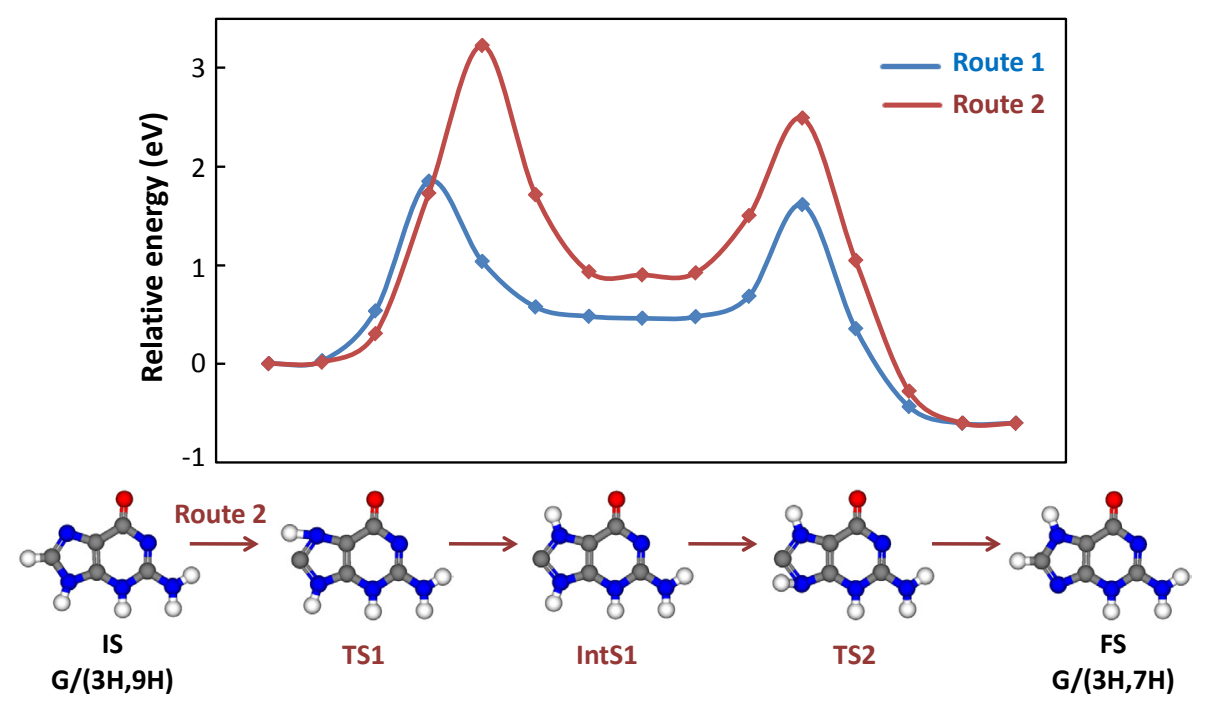

Figure S6. DFT calculated two reaction pathways for the second-step tautomerization from $\mathrm{G} /(3 \mathrm{H}, 9 \mathrm{H})$ to $\mathrm{G} /(3 \mathrm{H}, 7 \mathrm{H})$ in the gas phase involving a proton-transfer process from N9 to $\mathrm{N} 7$ where route 1 is found to be energetically most favorable and shown in the main text in Figure 3. The structural models of the local minima (IS, IntS1 and FS) and transition states (TS1 and TS2) along the reaction path (Route 2) are shown below the energy diagrams.
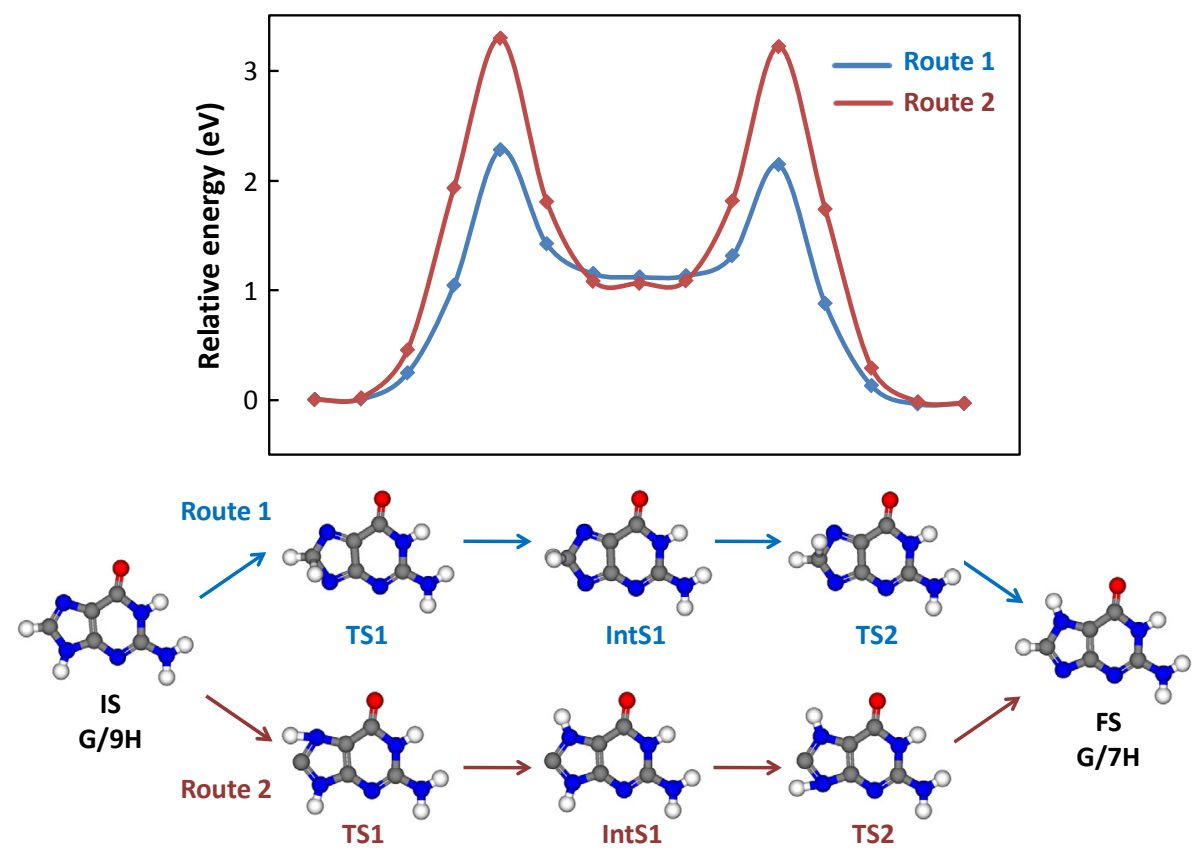

Figure S7. DFT calculated two reaction pathways for the first-step tautomerization from G/9H to $\mathrm{G} / 7 \mathrm{H}$ in the gas phase involving a proton-transfer process from N9 to N7. The structural models of the local minima (IS, IntS1 and FS) and transition states (TS1 and TS2) along different reaction paths (Route 1 and Route 2) are shown below the energy diagrams. 

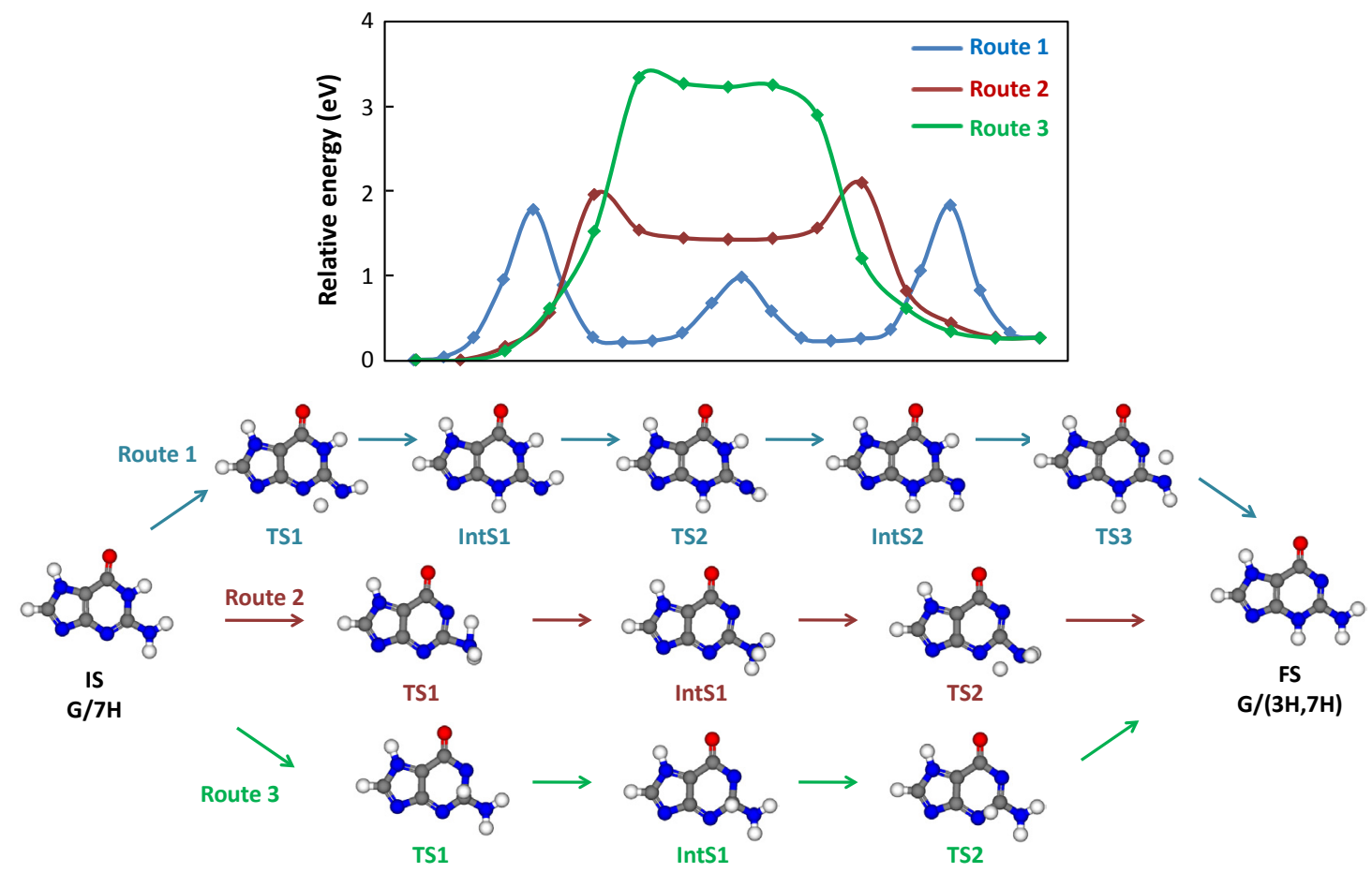

Figure S8. DFT calculated three reaction pathways for the second-step tautomerization from $\mathrm{G} / 7 \mathrm{H}$ to $\mathrm{G} /(3 \mathrm{H}, 7 \mathrm{H})$ in the gas phase involving a proton-transfer process from N1 to N3. The structural models of the local minima (IS, IntS1, IntS2 and FS) and transition states (TS1, TS2 and TS3) along different reaction paths (Route 1, Route 2 and Route 3) are shown below the energy diagrams.

Firstly, we have thought out the possible routes of tautomerization from $\mathrm{G} / 9 \mathrm{H}$ to $\mathrm{G} /(3 \mathrm{H}, 7 \mathrm{H})$ involving a double-proton-transfer process from N9 to N7 and from N1 to N3 without water molecules in the gas phase, where the sequence and corresponding routes need to be further determined. For the first pathway involving the tautomerization from $\mathrm{G} / 9 \mathrm{H}$ through $\mathrm{G} /(3 \mathrm{H}, 9 \mathrm{H})$ to $\mathrm{G} /(3 \mathrm{H}, 7 \mathrm{H})$, three routes for the first step have been taken into account as plotted in Figure $\mathrm{S} 5$ and Route 1 has the lowest energy barriers; two routes for the second step is plotted in Figure S6 and Route 1 has the lowest energy barriers. Accordingly, the most favorable pathway in this case is illustrated in Figure 3. Such proton-transfer processes from N1 to N3 have energy barriers of $1.91 \mathrm{eV}, 0.78 \mathrm{eV}$ and $1.75 \mathrm{eV}$ for proton-transfer from $\mathrm{NH}_{2}$ group to $\mathrm{N} 3$ site, flipping of the $\mathrm{N}-\mathrm{H}$ bond on $\mathrm{C} 2$ site, and proton-transfer from $\mathrm{N} 1$ site to $\mathrm{NH}$ group on $\mathrm{C} 2$ site, respectively. The N9-to-N7 proton-transfer processes have energy barriers of $1.85 \mathrm{eV}$ and $1.15 \mathrm{eV}$ for proton-transfer from N9 to C8 site and subsequent transfer from C8 to N7 site, respectively. Thus, the reaction barrier for the whole pathway is determined to be $1.91 \mathrm{eV}$.

For the second pathway from $\mathrm{G} / 9 \mathrm{H}$ through $\mathrm{G} / 7 \mathrm{H}$ to $\mathrm{G} /(3 \mathrm{H}, 7 \mathrm{H})$, similar routes for the two steps have been plotted in Figure S7 and S8 and the reaction barrier for the whole pathway is determined to be $2.28 \mathrm{eV}$, which is higher than that of the first pathway. Thus, this pathway is ruled out. 


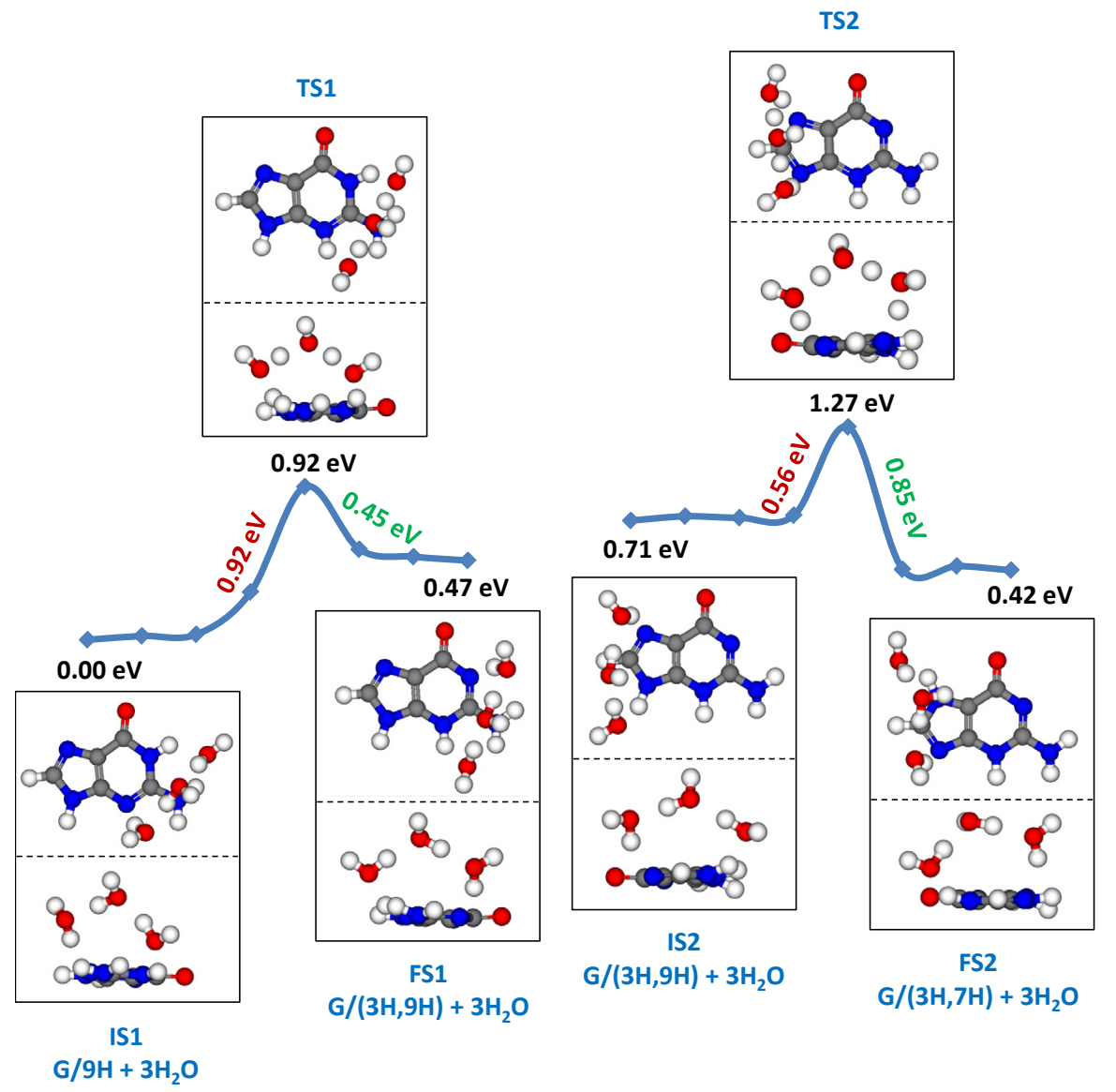

Figure S9. DFT calculated reaction pathway for the tautomerization from $\mathrm{G} / 9 \mathrm{H}$ to $\mathrm{G} /(3 \mathrm{H}, 7 \mathrm{H})$ with the assistance of water molecules. The pathway involves proton-transfer process from N1 to $\mathrm{N} 3$ forming the $\mathrm{G} /(3 \mathrm{H}, 9 \mathrm{H})$ intermediate with the assistance of three water molecules on the pyrimidine ring side as the first step and subsequent proton-transfer process from N9 to N7 forming the $\mathrm{FS} \mathrm{G} /(3 \mathrm{H}, 7 \mathrm{H})$ with three water molecules on the imidazole ring side. Energies of the local minima (IS1, FS1, IS2 and FS2) and transition states (TS1 and TS2) along the reaction path are given with respect to the initial state IS.

\section{References}

1. Hanus, M.; Ryjáček, F.; Kabeláč, M.; Kubař, T.; Bogdan, T. V.; Trygubenko, S. A.; Hobza, P. Correlated Ab Initio Study of Nucleic Acid Bases and Their Tautomers in the Gas Phase, in a Microhydrated Environment and in Aqueous Solution. Guanine: Surprising Stabilization of Rare Tautomers in Aqueous Solution. J. Am. Chem. Soc. 2003, 125, 7678-7688. 